valuuble. I refer to calculous affections of the kidneys, and certainly their effect in such cases is surprising. They act powerfully upon the kidneys, the small calculous deposits which find a nidus in their structure are mechanically washed away, and by their chemical agency upon the fluids of the body, they tend to remove that acid condition, which leads to such fearful results. As a remarkable proof of their value in such cases, I may mention what was said to me by $a$ lady five or six years ago, whoin I had recommended to drink them. "I have been at Vichy," she said, " every year, for six years, and in all that time I did not gain half the benefit which I have derived from the use of these waters for six weeks."

But I fear, gentlemen, I have already trespassed too much upon your time, and I hasten to bring this paper to a close.

I shall pass over the subject of the pure saline waters, with this one observation, that we find them a valuable remedy in delicate constitutions, where we wish to hare the effect of a mild laxative produced daily; and I come to notice lastly, the chalybeate, and the saline chalybeate springs. I consider these waters as being of at least as great importance in a therapeutic point of view, as the better known sulphur waters; but I need not occupy your time with any prolonged account of them, because, aware of their existence, you will readily perceive of how great an advantage they must prove to a large class of invalids.

The pure chalybeate springs are strong, containing about two grains and a half of iron in each gallon, which is held in solution by an excess of carbonic acid. I need say nothing of their therapeutic effects, with which you must be perfectly acquainted. But I am desirous of speaking in strong terms of the valuable remsedy which we have in the saline chalybeate waters. In these we have, in addition to the iron, a considerable quantity of muriate of soda, and of sulphate of soda. The effect of this is to act gently, but in most cases sufficiently, as an aperient; and we are thus enabled in this form, to administer with great benefit, a chalybeate in cases where it has long been indicated, but where, from a morbid excitability of the nervous system, or from other causes, it has been found impossible to continue its use for any length of time. I may also speak ir strong and even unqualified terıns, of the value of this water in a class of cases which are constantly met with in practice, where the secretions have become impuired or depraved, the bowels confined, the action of the liver sluggish, and all these symptoms depending upon a want of healthy tone and energy in the system, which, while it produces the symptoms, prevents that persevering continuance in the treatment which they appear to demand. In many other cases, into which your time will not allow me to enter, but which you will readily judge of, this water will be found a most veluable agent.

In the observations which I have had the honour to lay before you, I trust that I may not be thought to have unnecessarily occupied your time; I have been anxious to spread more widely through the country a knowledge of the valuable remedies which Harrogate affords: And to those aunong you, who know the place, or are in the habit of sending your patients there, I can confidently appeal for a confirmation of every word which I have uttered.
CASE OF ULCERATED STOMACH, CAUSING DEATH BY BEING SUIDDENLY DETACHED FROM ITS ADHESION TO THE PERITONEAL LINING OF THE ABDOMEN.

By Wrlliam Colnyns, Esq., Surgeon, Kenton.

(Read at the Annual Meeting of the South-Western District Branch of the Provincial Medical and Surgical Association.)

I was requested by a friend to open the body of a female relative, who had died suddenly under clrcumstances which he could not satisfactorily account for, and I extract from my case-book the following particulars which be gave me, and the result of the autopsy.

E. W., aged 21 , a very fine young woman, inclined to be corpulent, with a florid complexion, robust, and of active habits, had occasionally complained of rather acute pain in the left hypochondrium, after taking a full meal, but as it never lasted an hour at a time, and as her digestion was good, nothing was prescribed for her but some aperient pills. Being corpulent she was accustomed to have her stays laced very tight, and used to wear also a broad band round her waist, which was always made excessively tight, and it was thought the pressure might have occasioned the pain. One morning, after having used great exertion dancing all night at a ball, she eat a hearty breakfast, and quickly after walked out with some young friends. Suddenly she was seized with very severe pain in her left side, from which she said there was something tearing away; she shrieked violently, became faint, and fell down in the street; she was immediately removed to the house of her relative, which she had just left, efficient medical aid was instantly obtained, but after suffering intense agony for three hours, she expired.

Twelve hours after death I opened the abdominal cavity, there did not appear to be any omentum, but merely a ragged sort of fringe along the greater curvature of the stomach; there was no appearance of inflammation on the outer coat of the stomach or intestines ; but on that portion of the lesser curvature of the stomach anteriorly, which in the erect position of the body would have been in contact with the abdominal parictes on the left side, there was a considerable deposit of coagulable lymph, and a perforation through the coats of the stomach from internal ulceration, there being also a corresponding deposit of lymph on the inembranous lining of the abdominal cavity, to which it was apparent the stomach had adhered, and it was opposite this spot she had always complained of pain. The stomach was very large, and contained a good deal of undigested food, but the fluid parts had escaped into the cavity of the abdomen. All the other abdominal viscera were perfectly sound.

The stomach was produced for inspection.

\section{A CASE OF TETANUS SUCCESSFULLY TREATED.}

By William Gillard, Estg., Surgeon, Totness.

3 Read at the Annual Meeting of the South-Western District Branch of the Provincial Medical and Surgical Asociation.)

Deeember 28, 1838. R. L-, aged 20 years, a bealthy young man, received the whole charge of a gun in the front of the right thigh, about midway 